\title{
news
}

\section{news news news news news news news news}

\section{PFIZER LAUNCHES GEODON}

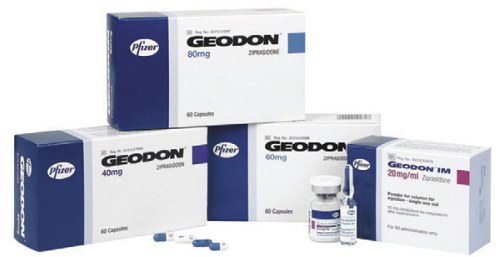

Pfizer Laboratories have launched Geodon, a new atypical antipsychotic for the treatment of schizophrenia.

Geodon has a unique combination of pharmacological activities, and demonstrates:

- predictable efficacy against both positive and negative symptoms in schizophrenia

- improvement of cognitive dysfunction and affective symptoms

- favourable tolerability and a healthy metabolic profile.

Switching to Geodon from currently available antipsychotic agents results in improvements in both positive and negative symptoms as well as improvements in symptoms of social impairment and cognition. Switching to Geodon also results in a significant reduction in weight and lipid levels, two important tolerability concerns with antipsychotic therapy.

Geodon is available as Geodon IM (ziprasidone mesylate) for intramuscular injection and in an oral formulation, Geodon Capsules (ziprasidone $\mathrm{HCl}$ ). The flexible dosage formulations, including $20 \mathrm{mg} / \mathrm{ml} \mathrm{IM}$ injection and 20, 40, 60 and $80 \mathrm{mg}$ capsules, facilitate the acute treatment of schizophrenia and the transition to long-term therapy while allowing a pharmacological continuum.

Geodon IM for injection and Geodon Capsules are available through Kinesis Logistics.

References available on request. Enquiries: Karin Keenan, Geodon Brand Manager, tel ( 011 ) 320-6000.

\section{DEPRESSION AFFECTS THE ELDERLY MORE THAN PHYSICAL ILLNESSES}

Depression appears to impair the quality of life of elderly individuals to a greater extent than chronic physical illness, say researchers who suggest that treating the condition may improve people's lives in spite of other medical conditions.

Hitchcock, Noël et al. reported their results in the Annals of Family Medicine (2004; 2: 555-562). The researchers collected data on 1801 individuals aged 60 years and older who met the criteria for major depression or dysthymia. The participants received either collaborative treatment or usual care for 12 months.

Collaborative care, which involved treatment with a depression care manager supervised by a psychiatrist and treatment with antidepressants or psychotherapy, resulted in $45 \%$ of patients achieving a $50 \%$ or greater reduction in depressive symptoms, compared with just $19 \%$ of those assigned to usual care.

Notably, the team found that depression was significantly associated with all four indicators of general health - physical functioning, mental health functioning, quality of life, and disability. As depression severity increased, quality of life and physical and mental health functioning declined, while disability increased.

This relationship remained significant after taking into account sociodemographic differences, other psychological dysfunction, and the presence of 11 chronic medical conditions, including chronic lung disease, hypertension, diabetes, arthritis, cancer, and heart disease.

The investigators further reported that despite the participants having an average of 3.8 chronic medical illnesses, depression severity made larger independent contributions to three of the four general health indicators than the medical co-morbidities.

\section{ONLINE SCHIZOPHRENIA ALGORITHM ALMOST READY}

An interactive, online schizophrenia treatment algorithm will provide a decision tree complete with supporting evidence and special clinical considerations for patients with co-morbid or pre-existing conditions, Herbert Meltzer (MD) said at the annual meeting of the European College of Neuropsychopharmacology.

The algorithm will be available by the beginning of 2005 at www.ipap.org and will be free to registered users.

The algorithm begins with a triage approach to classifying patients by pre-existing condition or co-morbid disorder. Clicking on those conditions - including emergent schizophrenia, substance abuse, suicidal and violent tendencies, non-compliance, and pregnancy - will link the user to information critical to the therapeutic decision-making process, Meltzer said.

The algorithm touches on both non-pharmacological and medical therapy. At each decision node, the user can access links to the supporting literature. The nodes also include information about cost-effectiveness of treatments.

Since the project is meant to have international application, each treatment node also takes into account the variability of international formularies and practice habits in different countries. The algorithm will be updated frequently with the newest evidence, Meltzer said. Users will receive automatic notifications of the updates, which are expected to occur quarterly. 